\title{
Beam Performance Optimization of Multibeam Imaging Sonar Based on the Hybrid Algorithm of Binary Particle Swarm Optimization and Convex Optimization
}

\author{
Weijie Xia, Xue Jin, and Fawang Dou \\ College of Electronic and Information Engineering, Nanjing University of Aeronautics and Astronautics, Nanjing 211106, China \\ Correspondence should be addressed to Xue Jin; 13236566268@163.com
}

Received 27 January 2016; Revised 8 April 2016; Accepted 27 April 2016

Academic Editor: Sotirios K. Goudos

Copyright (C) 2016 Weijie Xia et al. This is an open access article distributed under the Creative Commons Attribution License, which permits unrestricted use, distribution, and reproduction in any medium, provided the original work is properly cited.

It should be noted that the peak sidelobe level (PSLL) significantly influences the performance of the multibeam imaging sonar. Although a great amount of work has been done to suppress the PSLL of the array, one can verify that these methods do not provide optimal results when applied to the case of multiple patterns. In order to suppress the PSLL for multibeam imaging sonar array, a hybrid algorithm of binary particle swarm optimization (BPSO) and convex optimization is proposed in this paper. In this algorithm, the PSLL of multiple patterns is taken as the optimization objective. BPSO is considered as a global optimization algorithm to determine best common elements' positions and convex optimization is considered as a local optimization algorithm to optimize elements' weights, which guarantees the complete match of the two factors. At last, simulations are carried out to illustrate the effectiveness of the proposed algorithm in this paper. Results show that, for a sparse semicircular array with multiple patterns, the hybrid algorithm can obtain a lower PSLL compared with existing methods and it consumes less calculation time in comparison with other hybrid algorithms.

\section{Introduction}

The multibeam imaging sonar is becoming more and more popular for its high resolution in the applications of underwater detection [1]. The system may comprise an array of 100200 channels each associated with a hydrophone, amplifier, filter, and analog-to-digital converter (ADC), which results in considerable hardware complexity and computational load [2]. Consequently, it is meaningful to design a sparse array by selectively removing some elements, while keeping the same spatial aperture of the array. Once the number of elements and the spatial aperture have been fixed, the beam pattern of the sparse array, as compared with that of an equally spaced array, exhibits about the same mainlobe width but is characterized by much higher sidelobe level [3]. Thus beam performance optimization under multiple-pattern conditions is a priority. Here the design of the optimization model involves the peak sidelobe level (PSLL) suppression of multiple patterns for a sparse array, with the constraint of fixed number of elements and spatial aperture. Since the height of
PSLL depends on the element parameters (i.e., positions and weight coefficients), it is important to select both the best common elements' positions for multiple patterns and the best elements' weights for each single pattern of an unequally spaced array.

This is a high-dimensional nonlinear problem which has received more and more attention in the past thirty years [4]. A range of techniques have been proposed for sidelobe level suppression with single-pattern requirement, such as simulated annealing (SA) algorithm, genetic algorithm (GA), and particle swarm optimization (PSO) [5]. Some methods only determine optimal sidelobe-minimizing positions assuming the weights were constant $[6,7]$. In [8], the authors attempted to simultaneously optimize the weights and the positions of a linear array using SA algorithm. Recently, hybrid evolutionary algorithms were inspired by combining two algorithms together, which could suppress sidelobe level more effectively [9-11]. The work in [12] optimized elements' positions firstly and weight coefficients secondly, which resulted in a mismatch between the two factors. The work in 
[13] studied pattern synthesis of unequally spaced array based on the hybrid algorithm of GA and convex optimization, but GA has a very slow convergence speed in high-dimensional problems. What is more, these methods can not be easily extended to the case of multiple patterns since best elements' positions usually change with different patterns; thus the problem of minimizing the sidelobe level in the multibeam imaging sonar array has not been addressed yet.

PSO attracts more and more attention nowadays since it is easy to implement compared with other global optimization techniques. Binary particle swarm optimization (BPSO) [14] is used to solve discrete optimization problems in the binary search spaces. However, both PSO and BPSO may easily get trapped in local optima and show slow convergence rate when solving complex and high-dimensional multimodal objective functions. Motivated by the work in [13], a hybrid optimization algorithm of BPSO and convex optimization [15] is proposed in this paper, where convex optimization has strong local optimization capability. In our study, BPSO is used to determine optimal common elements' positions for multiple patterns globally, while convex optimization is used to optimize elements' weights for each pattern locally, which guarantees the complete match of the two factors and gets better optimization performance by reducing the dimension of particles in BPSO. Simulations demonstrate that, with the constraint of fixed number of elements, the proposed algorithm can effectively suppress the PSLL of sparse sonar array for multiple-pattern case while consuming less calculation time compared with other hybrid algorithms.

\section{Model of Optimization}

Let us consider the semicircular array of multibeam imaging sonar with $N$ elements, which are uniformly distributed shown in Figure 1. Assuming that the incident signal impinges upon the array with the arriving angle $\theta$ from the far field, the steering vector of the array is represented by

$$
\mathbf{G}(\theta)=\left[\begin{array}{c}
\exp \left(-\frac{j 2 \pi R \cos \left(\theta-\varphi_{1}\right)}{\lambda}\right) \\
\exp \left(-\frac{j 2 \pi R \cos \left(\theta-\varphi_{2}\right)}{\lambda}\right) \\
\vdots \\
\exp \left(-\frac{j 2 \pi R \cos \left(\theta-\varphi_{N}\right)}{\lambda}\right)
\end{array}\right],
$$

where $\varphi_{k}=(k-1) \pi /(N-1)$ for $k=1,2, \ldots, N$. In addition, $R$ is the radius of the array and $\lambda$ is the wavelength of the incident signal. Therefore, the response of the array is then given by

$$
P^{(l)}(\theta)=\sum_{k=1}^{N} w_{k}^{(l)} \exp \left(-\frac{j 2 \pi R \cos \left(\theta-\varphi_{k}\right)}{\lambda}\right),
$$

where $l=1,2, \ldots, L, L$ denotes the total number of the desired patterns and $w_{k}^{(l)}$ denotes the weight coefficient of the $k$ th element for the $l$ th pattern. We denote

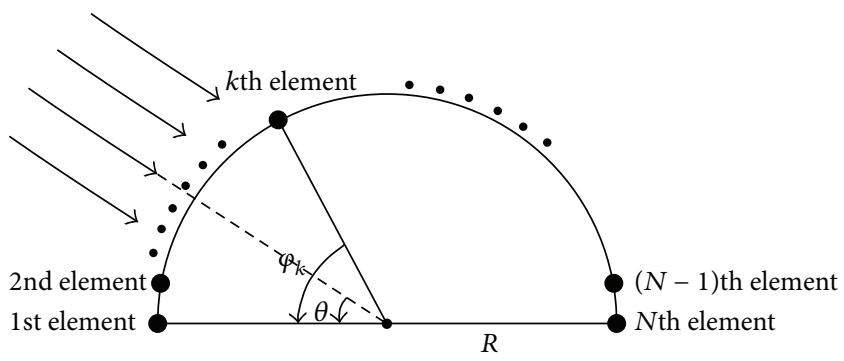

FIGURE 1: Diagram of the semicircular array.

$\mathbf{W}^{(l)}=\left[w_{1}^{(l)}, w_{2}^{(l)}, \ldots, w_{N}^{(l)}\right]$, and then the equation in (2) can be rewritten as

$$
P^{(l)}(\theta)=\mathbf{W}^{(l)} \mathbf{G}(\theta) .
$$

The problem considered here is to minimize the sidelobe level with multiple desired patterns in the sparse sonar array. The patterns include pencil beams with different mainlobe directions. For different patterns, the elements have optimized common positions but with probably different weight coefficients. The elements' positions can be expressed as the binary vector $\mathbf{X}_{1}=\left(x_{1}, x_{2}, \ldots, x_{N}\right)^{T}$. In order to keep the array aperture fixed, we must guarantee $x_{1}=1$ and $x_{N}=1$. The remaining components in $\mathbf{X}_{1}$ are either " 1 " (preserved) or " 0 " (removed). The real and the imaginary part of the weights can be expressed as $\mathbf{X}_{2}=\left(x_{N+1}, x_{N+2}, \ldots, x_{2 N}\right)^{T}, \mathbf{X}_{3}=\left(x_{2 N+1}\right.$, $\left.x_{2 N+2}, \ldots, x_{3 N}\right)^{T}$, respectively, where each component ranges from -1 to 1 . Thus the joint optimization variable is $\mathbf{X}=$ $\left[\mathbf{X}_{1}^{T}, \mathbf{X}_{2}^{T}, \mathbf{X}_{3}^{T}\right]^{T}$ and the problem can be formulated as

$$
\begin{array}{ll}
\min _{\mathbf{X}} & \text { PSLL } \\
\text { s.t. } & P^{(l)}\left(\theta_{d_{l}}\right)=1, \quad l=1,2, \ldots, L \\
& x_{1}=1, x_{N}=1 \\
& x_{2}, x_{3}, \ldots, x_{N-1}=0 \text { or } 1 \\
& -1 \leq x_{N+1}, x_{N+2}, \ldots, x_{3 N} \leq 1 .
\end{array}
$$

Here, "min" represents the abbreviation of "minimize" and "s.t." represents the abbreviation of "subject to." The first line of (4) involves the objective function needed to be minimized and the remaining four lines involve the constraints. In addition, $\theta_{d}$ denotes the mainlobe direction for the $l$ th pattern and PSLL denotes the peak sidelobe level for multiple patterns which is expressed as

$$
\text { PSLL }=\max _{l=1,2, \ldots, L}\left\{\max _{\substack{\theta_{S_{i}} \in \Omega_{l} \\ i=1,2, \ldots, I}}\left\{\left|\frac{P^{(l)}\left(\theta_{S_{i}}\right)}{P^{(l)}\left(\theta_{d_{l}}\right)}\right|\right\}\right\},
$$

where $\theta_{S_{i}}, i=1,2, \ldots, I$, are the sampling angles of the sidelobe region $\Omega_{l}$ for the $l$ th pattern. 


\section{Proposed Algorithm}

3.1. Binary Particle Swarm Optimization. Particle swarm optimization (PSO) is a population-based stochastic optimization algorithm proposed by Eberhart and Kennedy in 1995. It is inspired by social behavior of birds, which can effectively solve complicated optimization problems in the real search space.

A binary version of PSO, that is, binary particle swarm optimization (BPSO), was proposed to solve discrete optimization problems. What makes BPSO different from PSO is that each component of the particle position only has two possible values, "0" or " 1 ." In BPSO, the velocity and position updating equation can be expressed as

$$
\begin{aligned}
& v_{i d}^{t+1}=\omega * v_{i d}^{t}+c_{1} * \operatorname{rand}() *\left(p_{i d}^{t}-x_{i d}^{t}\right)+c_{2} \\
& * \operatorname{rand}() *\left(p_{g d}^{t}-x_{i d}^{t}\right) \text {, } \\
& x_{i d}^{t+!}= \begin{cases}1 & r_{i d}^{t}<S\left(v_{i d}^{t}\right) \\
0 & r_{i d}^{t} \geq S\left(v_{i d}^{t}\right),\end{cases}
\end{aligned}
$$

where $x_{i d}$ and $v_{i d}$, respectively, denote the $d$ th component of the position vector and velocity vector of the $i$ th particle, $p_{i d}$ denotes the $d$ th component of the personal optimal position of the $i$ th particle, and $p_{g d}$ denotes the $d$ th component of the global optimal position obtained so far by all particles. Besides, $\omega$ is a parameter known as the inertia weight, $c_{1}$ and $c_{2}$ are nonnegative constants called acceleration factors, $t$ denotes the current iteration, $t+1$ denotes the next iteration, $r_{i d}=\operatorname{rand}()$ represents a random number distributed between $(0,1)$, and $S(x)$ denotes Sigmoid function given by

$$
S(x)=\frac{1}{1+e^{-x}} .
$$

Considering that the velocity of the particles is often constrained within $\left[-v_{\max }, v_{\max }\right], S\left(v_{i d}^{t}\right)$ is especially described as

$$
S\left(v_{i d}^{t}\right)= \begin{cases}\frac{1}{1+e^{v_{\max }}} \longrightarrow 0 & v_{i d}^{t}=-v_{\max } \longrightarrow-\infty \\ 0.5 & v_{i d}^{t}=0 \\ \frac{1}{1+e^{-v_{\max }}} \longrightarrow 1 & v_{i d}^{t}=v_{\max } \longrightarrow \infty \\ \frac{1}{1+e^{-v_{i d}^{t}}} & \text { else. }\end{cases}
$$

Thus $S\left(v_{i d}^{t}\right)$ varies between $\left[1 /\left(1+e^{v_{\max }}\right), 1 /\left(1+e^{-v_{\max }}\right)\right]$, which is a subset of $[0,1]$.

In this paper, BPSO is adopted to determine the best elements' positions of the sparse sonar array with elements placed at optional positions. The first position and last position are always occupied and the rest of the positions are uncertain. The fitness function has only one term that aims at minimizing the PSLL for multiple patterns according to (5).

3.2. Convex Optimization. Convex optimization refers to the minimization of a convex objective function subject to convex constraints. Since the joint optimization variable is very high-dimensional, the improved BPSO mixed with the convex optimization is proposed to get better optimization performance by reducing the dimension of particles. For each particle, the weights for each pattern can be optimized by convex optimization when computing fitness value, on the premise that the elements' positions $x_{1}, x_{2}, \ldots, x_{N}$ are selected by BPSO. For different patterns, the elements have optimized common positions but with probably different weight coefficients. Thus the optimization subproblem in the fitness function can be formulated as

$$
\begin{aligned}
& \text { for } \quad l=1: L \\
& \min \max _{\substack{\theta_{S_{i}} \in \Omega_{l} \\
i=1,2, \ldots, I}}\left|\mathbf{W}^{(l)} \mathbf{G}\left(\theta_{S_{i}}\right)\right| \\
& \text { s.t. } \quad \mathbf{W}^{(l)} \mathbf{G}\left(\theta_{d_{l}}\right)=1 \\
& y=\max _{\substack{\theta_{S_{i}} \in \Omega_{l} \\
i=1,2, \ldots, I}}\left\{\left|\frac{\mathbf{W}^{(l)} \mathbf{G}\left(\theta_{S_{i}}\right)}{\mathbf{W}^{(l)} \mathbf{G}\left(\theta_{d_{l}}\right)}\right|\right\} \\
& \text { end } \\
& \text { PSLL }=\max _{l=1, \ldots, L}(y),
\end{aligned}
$$

where $\theta_{S_{i}}, i=1,2, \ldots, I$, are the sampling angles of the sidelobe region for the $l$ th pattern and $\theta_{d_{l}}$ denotes the mainlobe direction for the $l$ th pattern.

As is known to all, formulation (9) is a convex problem that can be solved by SeDuMi or CVX of the Matlab toolbox effectively, which is helpful to save the calculation time of the hybrid algorithm. When the optimization subproblem is solved, the best weight coefficients for the lth pattern are $\mathbf{W}^{(l)}=\left[w_{1}^{(l)}, w_{2}^{(l)}, \ldots, w_{N}^{(l)}\right], l=1,2, \ldots, L$. Then the peak sidelobe level for multiple patterns is taken as the return value of the fitness function for each particle according to (5).

3.3. Procedure of the Hybrid Algorithm. In this paper, the hybrid algorithm of BPSO and convex optimization is proposed to optimize the beam performance, especially minimizing the sidelobe level of the sparse sonar array. BPSO is applied as global optimization algorithm to optimize the common elements' positions and then convex optimization is applied as local optimization algorithm to optimize the weights when computing fitness value. All these guarantee that the particles can search the whole space with little possibility of falling into the local optimal solution. It is worth noting that the element number of the sparse array is fixed during the update of the particles' positions; that is to say, the number of components that are "1" for each particle position should be unchangeable. If it varies, a few components will be randomly set to " 1 " or " 0 ." The procedure of the proposed algorithm is shown in Figure 2. Finally, the hybrid algorithm will converge to a set of common elements' positions and multiple sets of weight coefficients with fixed number of elements.

\section{Evaluations}

For the multibeam imaging sonar, let us consider the semicircular array with 120 elements placed at 180 optional 
TABLE 1: PSLL and calculation time of different methods.

\begin{tabular}{lcc}
\hline Method & PSLL (dB) & Calculation time (hour) \\
\hline BPSO (only optimize positions) & -7.4894 & - \\
BPSO with PSO (optimize positions firstly and weights secondly) & -16.0519 & 73.8 \\
Hybrid algorithm of genetic algorithm and convex optimization & -19.5392 & 16.5 \\
Proposed algorithm in the paper & -22.1643 & 7.5 \\
\hline
\end{tabular}

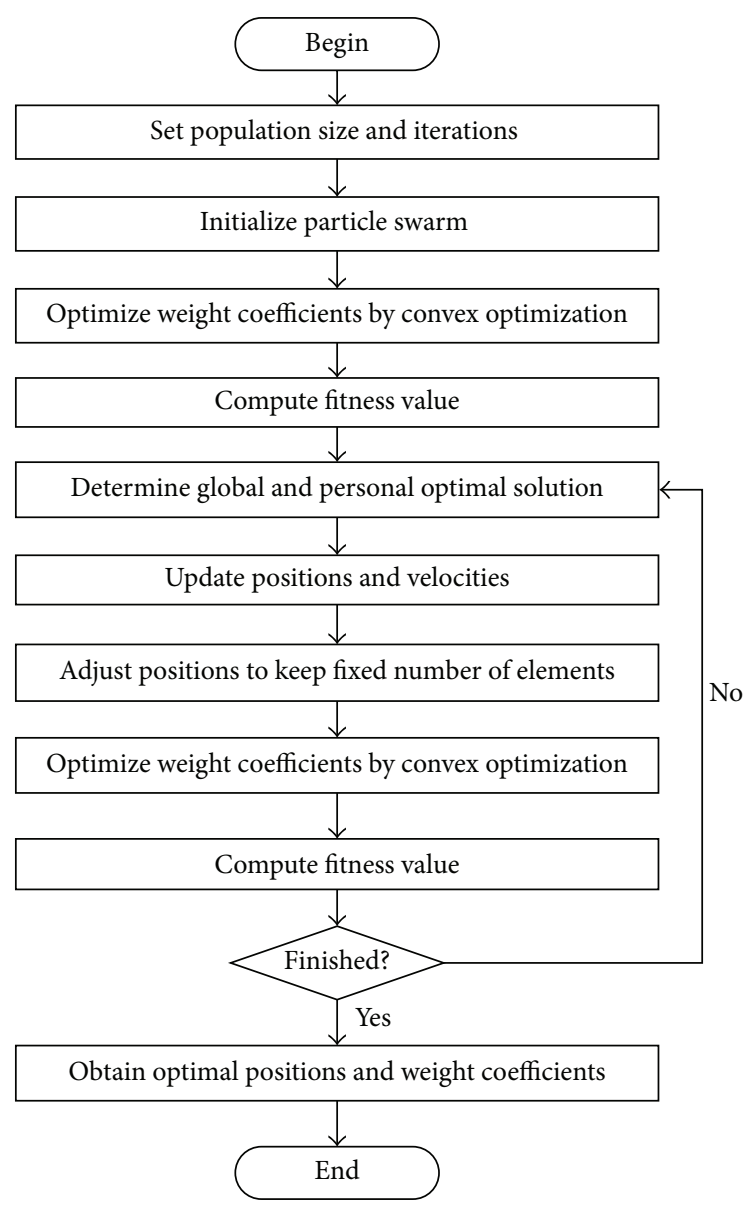

FIGURE 2: Procedure of the proposed algorithm.

positions, and the array is required to generate 538 beam patterns. The patterns include pencil beams whose mainlobe directions are uniformly distributed in the steering region, that is, $\left[45^{\circ}, 135^{\circ}\right]$. The radius of the array is $0.12 \mathrm{~m}$ and the wavelength of the incident signal is $0.0033 \mathrm{~m}$.

In the BPSO, a population pool including 100 particles is initialized and the maximum number of runs is set equal to 100. The parameter values are chosen as $\omega=1, c_{1}=c_{2}=2$ for the search process and the maximum velocity is set as $v_{\max }=6.0$. Figure 3 shows the beam pattern that is optimized by the proposed algorithm in this paper. It can be seen that the PSLL of multiple patterns can be suppressed to $-22.1643 \mathrm{~dB}$. Figure 4 shows the elements' distribution of the sparse array obtained by the proposed algorithm. The behavior of weight coefficients is significantly difficult to depict here because

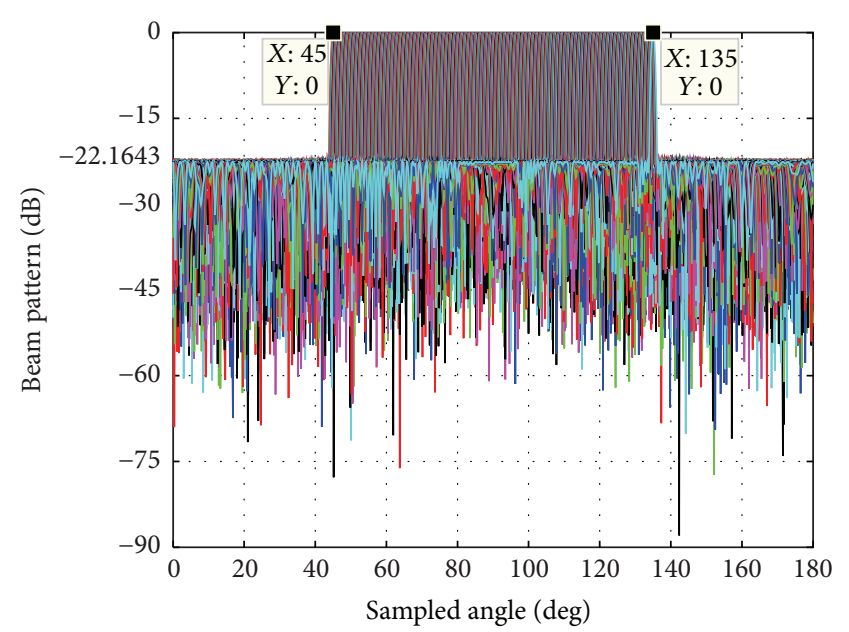

FIGURE 3: Beam pattern optimized by the proposed algorithm.

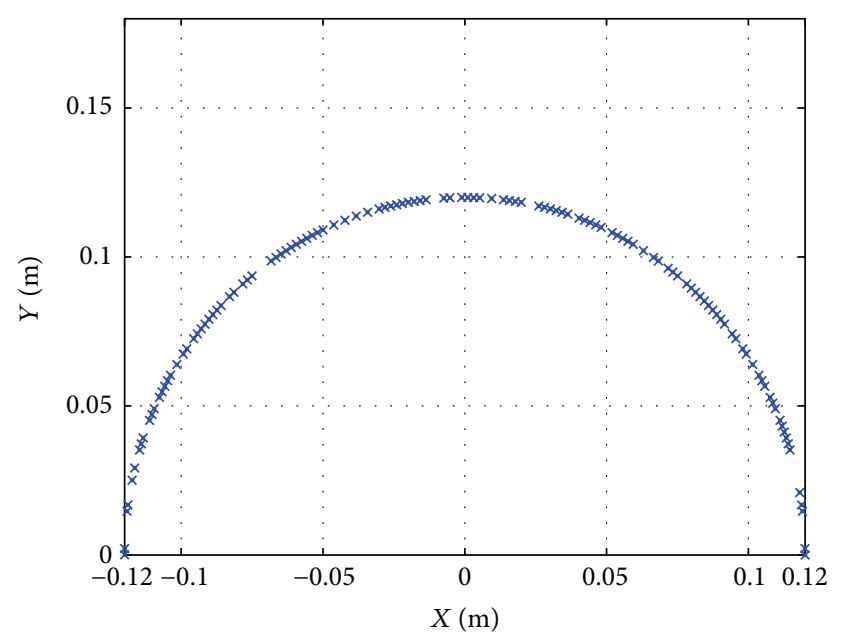

FIGURE 4: Elements' distribution of the proposed algorithm.

the algorithm obtains multiple sets of weights for multiple patterns.

For comparison, we apply the methods in $[6,12,13]$ to multiple-pattern conditions and give the obtained results in Table 1 . In the GA, the population size and the maximum number of runs are both set equal to 100. The crossover probability is set as 0.6 and the mutation probability is set as 0.04 . The beam patterns are, respectively, visualized in Figures 5, 6, and 7. The average convergence curves of the proposed method and the other three methods discussed above are depicted in Figure 8. It demonstrates that the proposed 


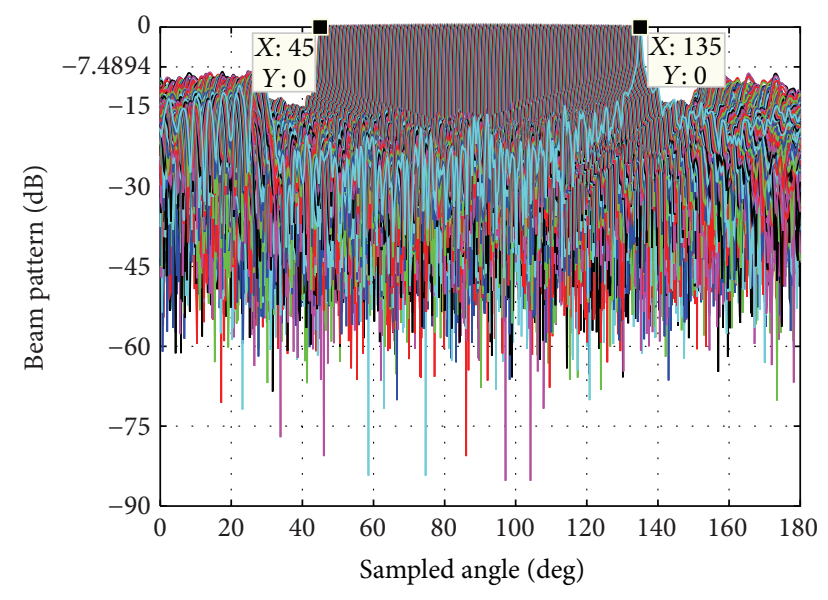

FIGURE 5: Beam pattern by only optimizing elements' positions.

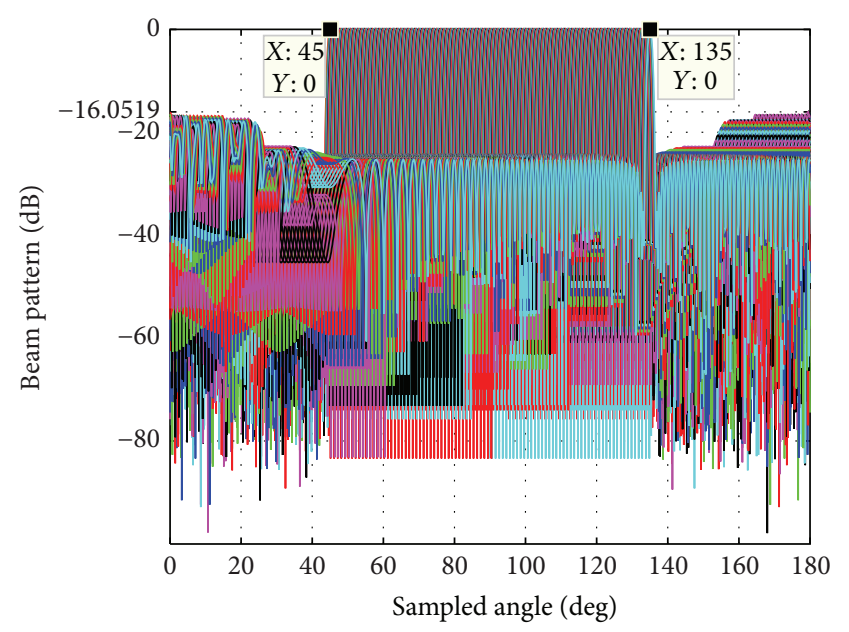

FIGURE 6: Beam pattern optimized by BPSO firstly and PSO secondly.

algorithm has excellent global optimization performance and fast convergence speed.

In conclusion, the simulations demonstrate the effectiveness of the proposed optimization algorithm based on BPSO and convex optimization. Different from prior work in [6$8,12,13]$, the algorithm proposed in this paper considers both elements' positions and weights during the PSLL suppression and keeps the complete match of the two factors. It is more important that the algorithm is applicable to the case of multiple patterns and can obtain a lower PSLL while consuming less calculation time compared with other methods based on the idea in prior literature.

\section{Conclusion}

In this paper, a hybrid optimization algorithm of BPSO and convex optimization is proposed to suppress the peak sidelobe level of sparse array for the multibeam imaging

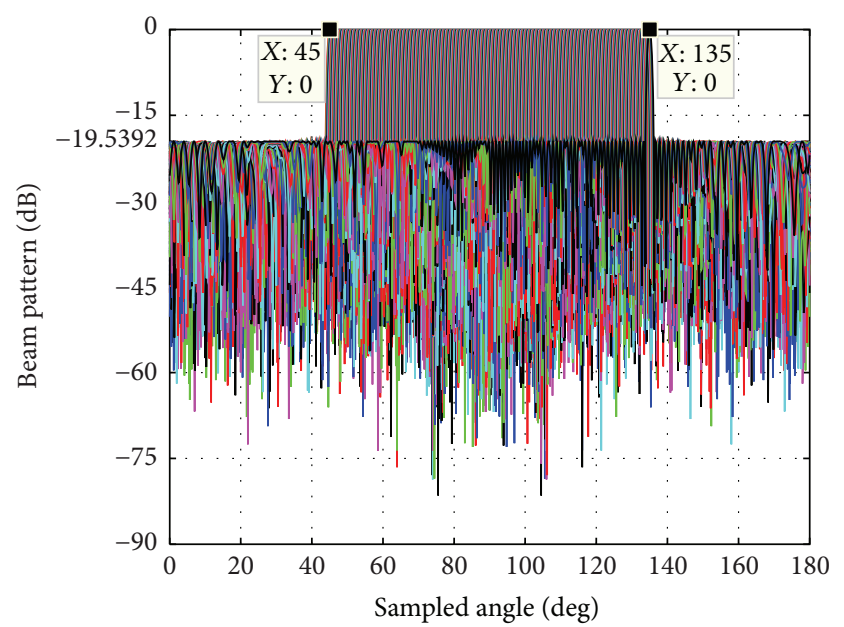

FIGURE 7: Beam pattern optimized by the hybrid algorithm of GA and convex optimization.

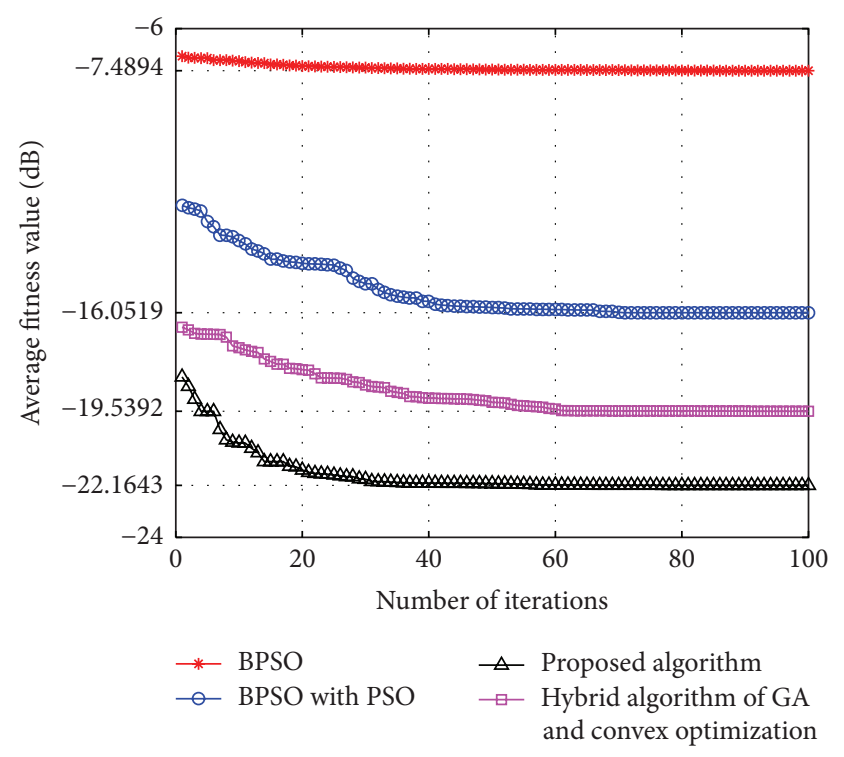

FIgURE 8: Convergence curves of different methods.

sonar. In this algorithm, BPSO is applied to determine optimal common elements' positions for multiple patterns and convex optimization is to optimize the elements' weights for each single pattern. The results show that, for the sparse semicircular array with 120 elements placed at 180 optional positions, the PSLL can be suppressed to $-22.1643 \mathrm{~dB}$ by the proposed algorithm, which is lower than those reported in literatures, and the calculation time is significantly decreased. Thus the work here presents excellent optimization performance for multibeam imaging sonar.

\section{Competing Interests}

The authors declare that there is no conflict of interests regarding the publication of this paper. 


\section{Acknowledgments}

The work was supported by the Production-Study-Research Prospective Joint Research Project of Jiangsu Province of China (Grant no. BY2014003-14) and the Priority Academic Program Development of Jiangsu Higher Education Institutions.

\section{References}

[1] L.-Y. Weng, M. Li, Z. B. Gong, and S. G. Ma, "Underwater object detection and localization based on multi-beam sonar image processing," in Proceedings of the IEEE International Conference on Robotics and Biomimetics (ROBIO '12), pp. 514519, Guangzhou, China, December 2012.

[2] A. E. Albright Blomberg, A. Austeng, R. E. Hansen, and S. A. V. Synnes, "Improving sonar performance in shallow water using adaptive beamforming," IEEE Journal of Oceanic Engineering, vol. 38, no. 2, pp. 297-307, 2013.

[3] J. W. Hooker and R. K. Arora, "Optimal thinning levels in linear arrays," IEEE Antennas and Wireless Propagation Letters, vol. 9, pp. 771-774, 2010.

[4] T. Isernia, A. Massa, A. F. Morabito, and P. Rocca, "On the optimal synthesis of phase-only reconfigurable antenna arrays," in Proceedings of the 5th European Conference on Antennas and Propagation (EUCAP '11), pp. 2074-2077, April 2011.

[5] E. Ozcan and C. K. Mohan, "Particle swarm optimization: surfing the waves," in Proceedings of the Congress on IEEE Evolutionary Computation (CEC '99), vol. 3, pp. 6-9, Washington, DC, USA, July 1999.

[6] M. M. Khodier and C. G. Christodoulou, "Sidelobe level and null control using particle swarm optimization," IEEE Transactions on Antennas and Propagation, vol. 53, no. 8, pp. 2674-2679, 2005.

[7] S. K. Goudos, V. Moysiadou, T. Samaras, K. Siakavara, and J. N. Sahalos, "Application of a comprehensive learning particle swarm optimizer to unequally spaced linear array synthesis with sidelobe level suppression and null control," IEEE Antennas and Wireless Propagation Letters, vol. 9, pp. 125-129, 2010.

[8] V. Murino, A. Trucco, and C. S. Regazzoni, "Synthesis of unequally spaced arrays by simulated annealing," IEEE Transactions on Signal Processing, vol. 44, no. 1, pp. 119-123, 1996.

[9] Y.-Y. Bai, S. Xiao, C. Liu, and B.-Z. Wang, "A hybrid IWO/PSO algorithm for pattern synthesis of conformal phased arrays," IEEE Transactions on Antennas and Propagation, vol. 61, no. 4, pp. 2328-2332, 2013.

[10] W. T. Li, X. W. Shi, Y. Q. Hei, S. F. Liu, and J. Zhu, "A hybrid optimization algorithm and its application for conformal array pattern synthesis," IEEE Transactions on Antennas and Propagation, vol. 58, no. 10, pp. 3401-3406, 2010.

[11] Y. Fan, R. H. Jin, Z. Y. Wu, B. Liu, and J. P. Geng, "Pattern synthesis of linear arrays using a hybrid optimization algorithm," in Proceedings of the 7th International Conference on Signal Processing Proceedings (ICSP '04), vol. 1, pp. 428-430, Beijing, China, September 2004.

[12] X.-Z. Liu, W.-L. Yang, Z.-Z. Gao, and X.-D. Zhang, "A new method for the synthesis of sparse linear array," in Proceedings of the IEEE CIE 6th International Conference on Radar (RADAR '11), pp. 1139-1142, October 2011.

[13] T. Bian and Y. Zhang, "Synthesis of unequally spaced array by genetic algorithm and convex optimization," in Proceedings of the International Radar Conference IET, vol. 120, pp. 1-4, Guilin, China, April 2009.

[14] Z. Beheshti, S. M. Shamsuddin, and S. Hasan, "Memetic binary particle swarm optimization for discrete optimization problems," Information Sciences, vol. 299, pp. 58-84, 2015.

[15] S. Boyd and L. Vandenberghe, Convex Optimization, Cambridge University Press, Cambridge, Uk, 2004. 


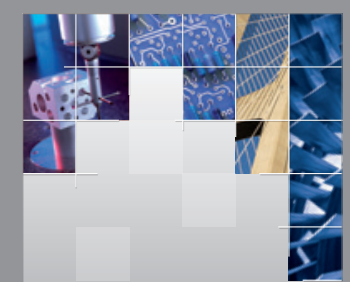

\section{Enfincering}
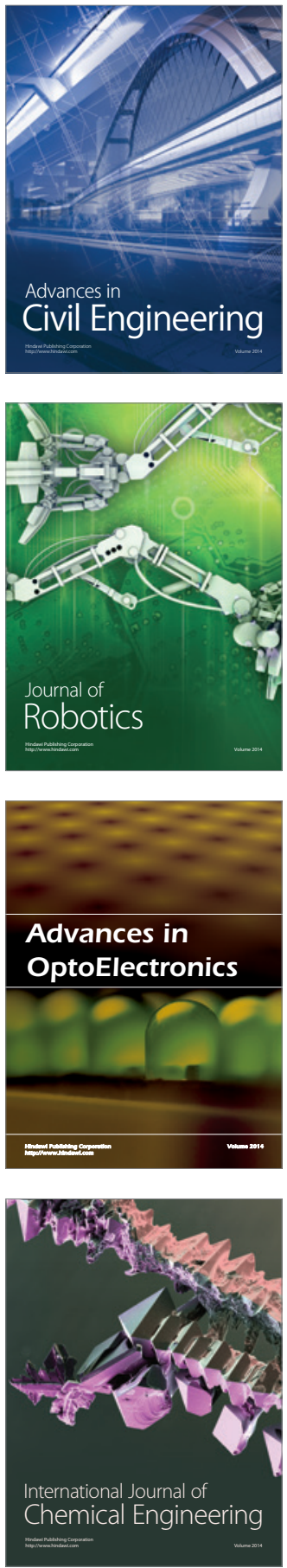

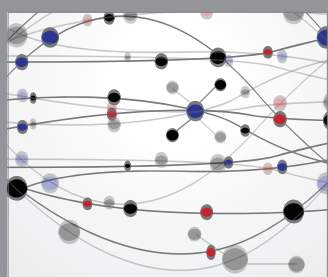

The Scientific World Journal

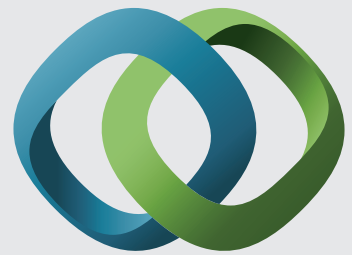

\section{Hindawi}

Submit your manuscripts at

http://www.hindawi.com
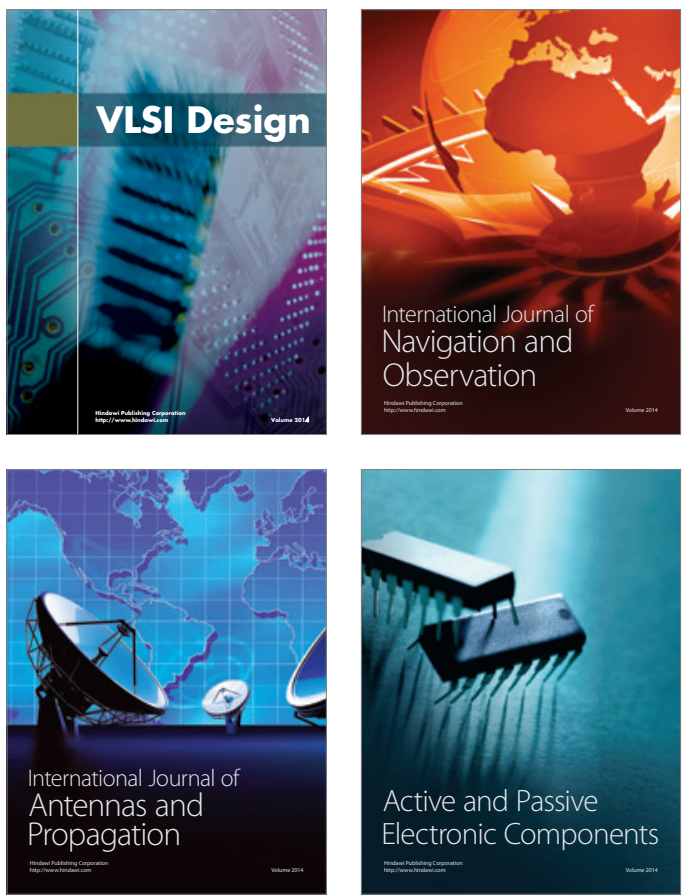
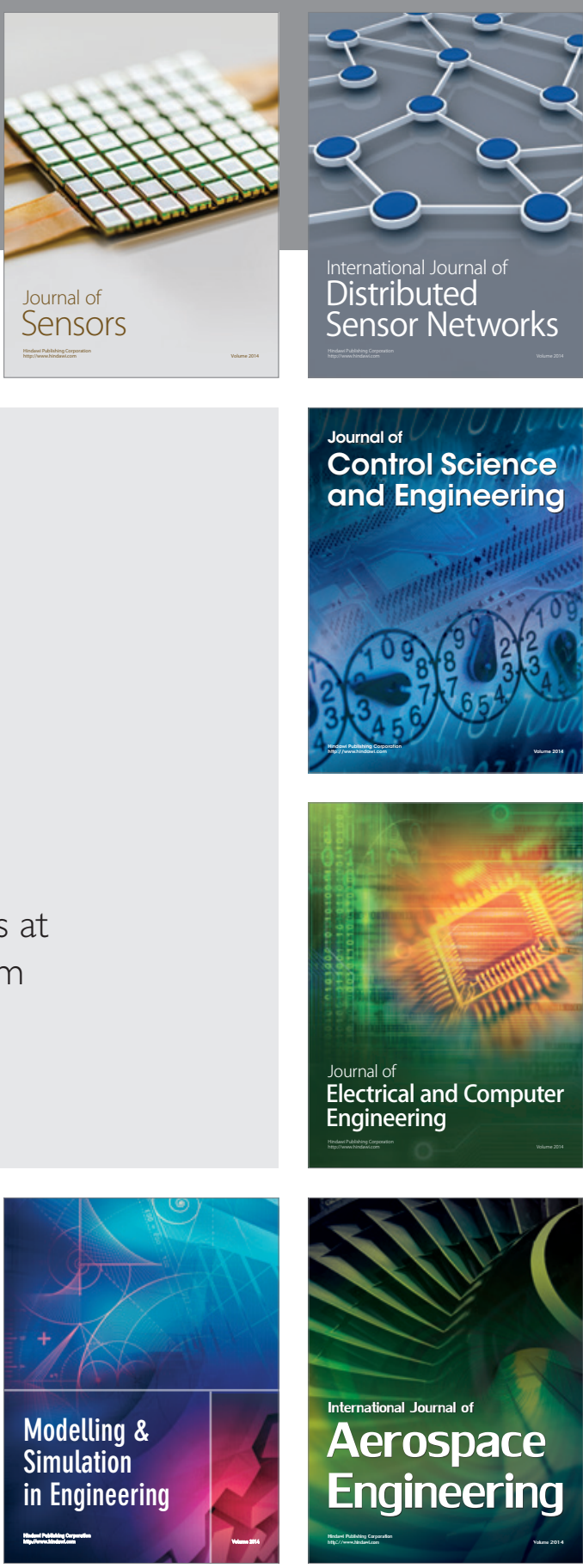

International Journal of

Distributed

Sensor Networks

Journal of

Control Science

and Engineering
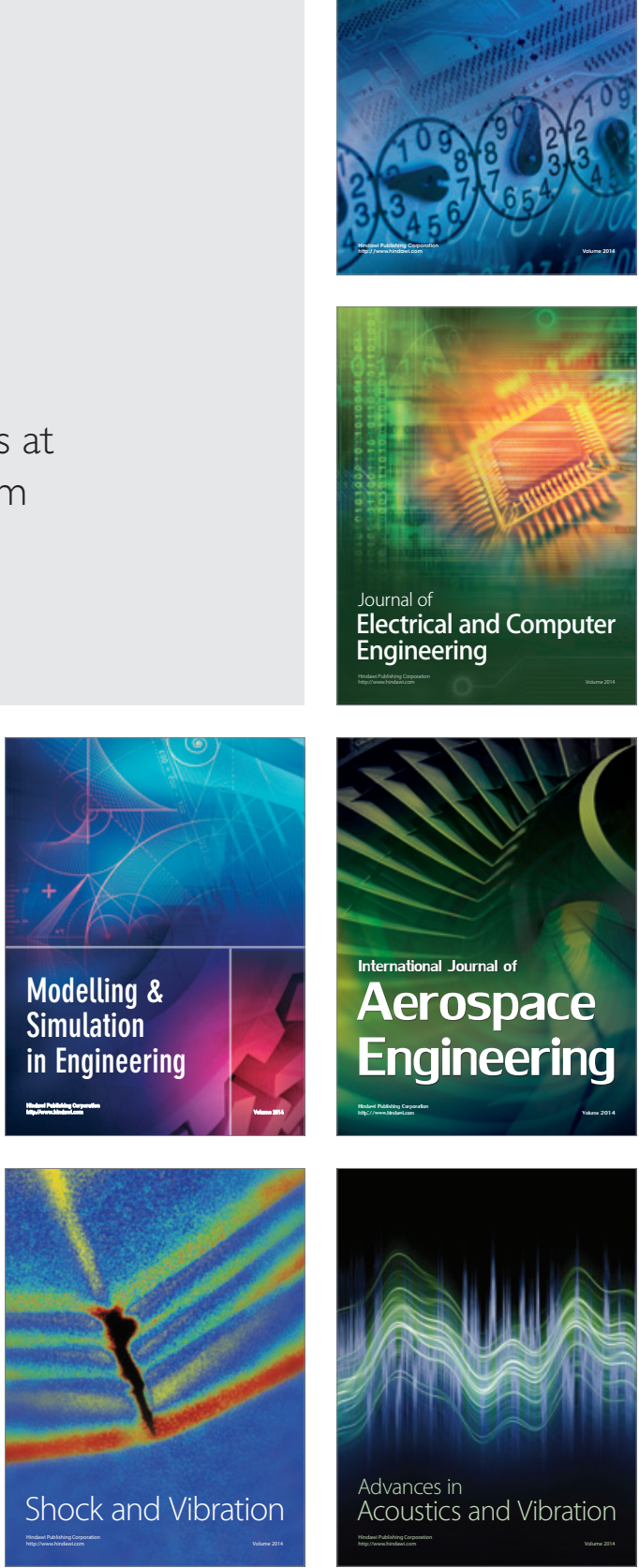